\title{
Entrelacs
}

Cinéma et audiovisuel

15 | 2018

Récits de soi

\section{Récits du nous}

\section{Catherine Guéneau et Gérard Leblanc}

\section{(2) OpenEdition \\ Journals}

Édition électronique

URL : http://journals.openedition.org/entrelacs/2991

DOI : 10.4000/entrelacs.2991

ISBN : 2261-5482

ISSN : 2261-5482

Éditeur

Éditions Téraèdre

Référence électronique

Catherine Guéneau et Gérard Leblanc, « Récits du nous », Entrelacs [En ligne], 15 | 2018, mis en ligne le 21 octobre 2018, consulté le 30 avril 2019. URL : http://journals.openedition.org/entrelacs/2991 ;

DOI : 10.4000/entrelacs.2991

Ce document a été généré automatiquement le 30 avril 2019.

Tous droits réservés 


\title{
Récits du nous
}

\author{
Catherine Guéneau et Gérard Leblanc
}

1 La sollicitation de Claire Chatelet et de Julie Savelli nous parvient alors que nous sommes en train d'écrire. Non pour un film, pour un livre. Un livre que nous écrivons à deux. À deux, comme nous avons réalisé nos films (22 depuis 2001 avons-nous calculé récemment, plus ou moins courts, plus ou moins longs). Ce livre tourne autour du thème de la "parentalité" auquel nous avions déjà « sacrifié » lorsque Lucas, notre enfant unique, était bébé. C'est ainsi que notre deuxième film en commun Premiers mois (2005) était né. Et notre deuxième projet de livre, également en cours d'écriture, tourne autour du thème de la relation amoureuse qui avait déjà fourni matière à la réalisation de notre premier film en commun En amour (2001). Au cours de toutes ces années, comme on peut le constater, nous n'avons guère changé de thèmes ou plutôt de " vécus » même si, dans l'intervalle, nous avons réalisé aussi des films qui ne relèvent pas de l'autobiographie (nous y reviendrons).

\section{Je, nous, je}

2 Aurions-nous changé de moyen d'expression? Pas vraiment. Nous avons toujours désiré alterner le cinéma et la littérature. Nous n'avons jamais ressenti ces deux moyens d'expression comme exclusifs l'un de l'autre (surtout lorsque la littérature vire à la poésie). Il y a de la littérature dans nos films et il y a du cinéma dans notre écriture.

Voyons d'abord ce qu'ils ont en commun dans nos projets expressifs.

3 Je n'est pas je quand on travaille à deux. Il s'agit de s'exprimer en interaction avec l'autre, à la recherche d'une nouvelle identité qui ne pourrait, ni se concevoir, ni se construire en dehors de cette interaction. Il est question d'inventer quelqu'un qui n'existe pas. Cette orientation ne relève pas d'une contrainte mais de la conviction qu'aucun être humain ne se suffit à lui-même. Chacun(e) a besoin d'un(e) autre pour vivre au plus aigu de soi. La rencontre avec l'autre culmine évidemment dans la relation amoureuse.

4 Qu'il s'agisse d'écrire ou de filmer, l'élaboration de l'œuvre sera dialogique, non au sens défini par Mikhail Bakhtine (le multiple dans l'un) mais au sens d'une libération du 
multiple dans chacun(e). Cette posture dialogique peut intégrer des tiers (un bébé dans Premiers mois ou encore Diderot dans notre plus récent Langres, Diderot et nous..., 2014). Dans tous les cas de figure, il ne s'agira pas d'additionner des subjectivités mais de les transformer les unes au contact des autres.

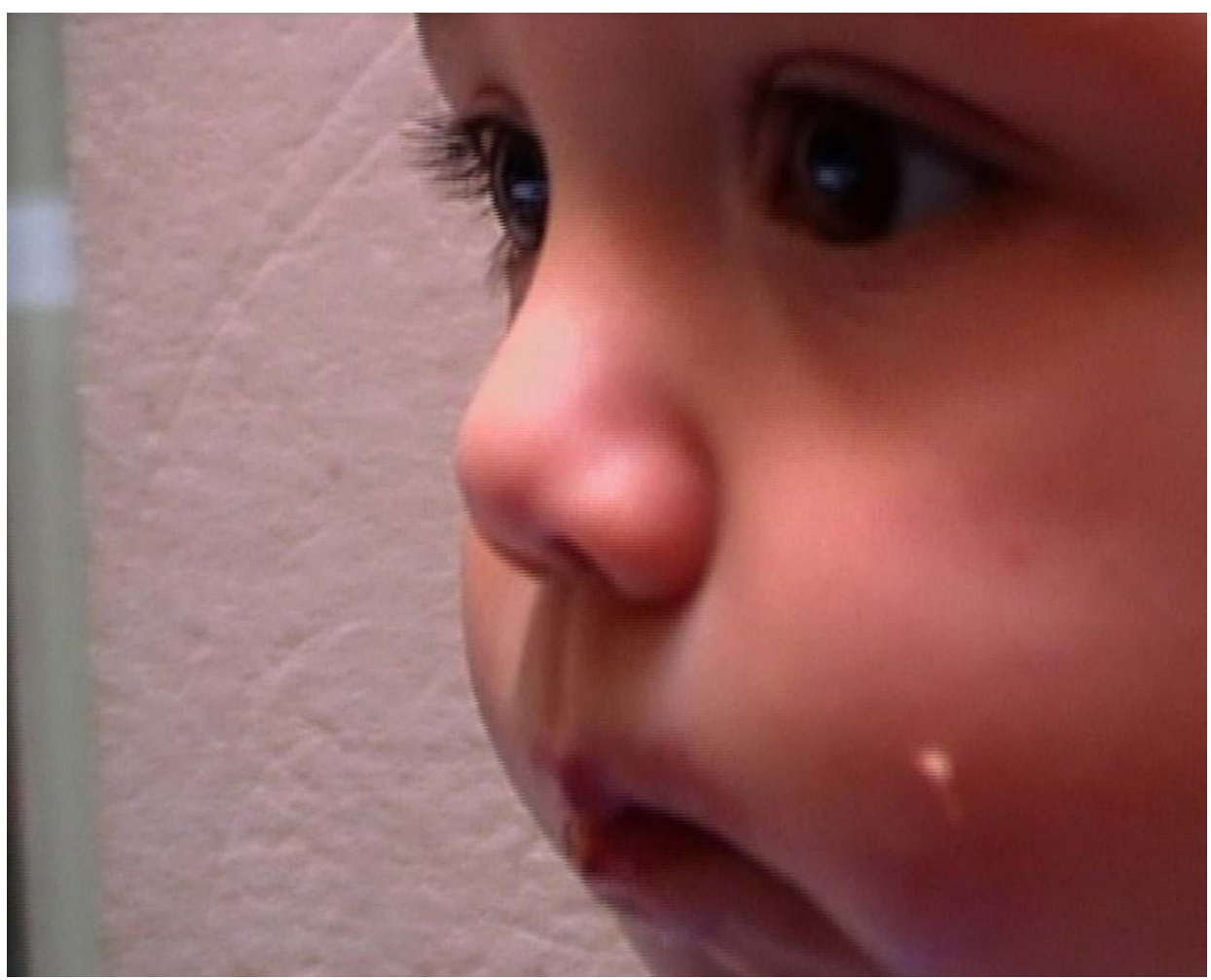

Premiers mois, 2005

5 On objectera peut-être qu'il ne saurait être question de transformer la subjectivité d'un mort, Diderot en l'occurrence. Dans Langres, Diderot et nous..., nous faisons jouer aux ciels et à l'environnement minéral un rôle qui excède celui qu'ils jouent réellement dans l'œuvre de Diderot. Nous invitons le lecteur - le spectateur - à réévaluer leur importance dans la subjectivité de l'auteur et dans sa trajectoire de philosophe. La subjectivité d'un auteur est toujours en mouvement tant qu'il reste vivant en tant qu'auteur'. De la même façon, la relecture de Diderot nous fait bouger subjectivement, elle instaure un nouveau dialogue avec lui. Le montage de notre film est ordonné tout entier autour de son mot d'ordre maintes fois réitéré: « le premier devoir de l'homme est de se rendre heureux ». Il est plus facile de dialoguer avec des morts qui restent vivants qu'avec des vivants qui sont déjà morts.

6 Cette conception particulière du dialogue consiste à passer d'une identité close (y compris multiple) à une identité ouverte où les interactions externes jouent un rôle déterminant.

7 Mais si je n'est plus je, est-ce un nous? À part le nous féodal de majesté, dont les institutions se repaissent encore aujourd'hui (combien de thésards se croient obligés d'écrire nous pour dire je), le nous peut être aussi une extension du je. Il arrive fréquemment que le nous soit un je masqué. Il s'autorise de l'approbation des autres pour s'énoncer de façon plus massive, assertive. Le nous est alors un je englobant. Nous pensons que... mais c'est toi qui contrains un nous fictif à penser comme toi. 
8 Le nous réel est un nouveau je qui se constitue à partir des interactions entre les différents je. Le je ne s'oppose plus au nous, pas davantage qu'il ne l'englobe. Les deux pronoms entrent en équivalence.

$9 \quad$ Faut-il dire je?

Faut-il dire nous?

Nous avons fait le pari de la création possible d'une nouvelle identité.

Ni toi ni moi.

Quelqu'un d'autre.

À construire

Est-ce une collaboration?

Pas vraiment.

C'est une double mise en jeu.

10 Mais cette mise en jeu ne correspond-t-elle pas à l'acte de création lui-même qui consiste toujours, loin de tout fantasme de maitrise (le je origine), à découvrir un autre en soi ? Tout processus de création produit de nouveaux je, de nouveaux nous.

\section{Cinéma, littérature}

11 Construire un nous en littérature et en cinéma, est-ce pourtant du pareil au même?

Les conditions matérielles de création semblent très différentes. En littérature, chacun écrit de son côté, avec ses mots, avec son style. En cinéma, les deux auteurs peuvent croiser leurs écritures pour élaborer des hypothèses de scénario, de filmage et en discuter. Mais ensuite ils se retrouvent ensemble. Au filmage comme au montage. Ils effectuent les différents choix en commun. Le cinéma semble plus à même de favoriser des interactions que la littérature.

Les images se mêlent, ne nous appartiennent plus, qui a filmé quoi ? On finit par ne plus le savoir. Peu importe. Ce qui importe, c'est le devenir de ces images dans notre film, leurs interactions. Ton regard s'ajoute au mien en le démultipliant. Lâcher prise et sortir de son propre enfermement identitaire. Comme dans la vie, me confronter à toi en permanence pour sans cesse me découvrir autre, «toucher un point en moi auparavant inaccessible». Former un troisième terme à partir des deux premiers. 1+1=3. La littérature serait-elle moins propice que le cinéma pour le travail en commun ? Nous avons découvert récemment une forme de dialogue littéraire très productif, à l'intérieur duquel nous confrontons au quotidien nos textes. Une autre façon d'interagir, d'assembler des mots comme nous le faisions avec les sons et les images, de contrer le travail solitaire de l'écriture.

Travailler de cette manière c'est aussi augmenter la part d'imprévisible de l'écriture. Chaque jour, un peu comme une dégustation à l'aveugle, un texte inédit à découvrir, une stimulation renouvelée, relancée par tes mots face aux miens. L'écriture va se construire au coeur de l'altérité, comme pour mieux métisser notre texte.

Mais si la construction dialogique existe de façon plus décalée en littérature, elle n'en contribue pas moins à forger un nous. Dialoguer ne consiste pas à confronter des sensations, des analyses, des positions mais à les mettre en jeu dans la lecture de l'autre qui les interprète et les traduit dans sa propre écriture. Je écrit dans l'écriture de l'autre et réciproquement. Comme si cette autre écriture lisait ce qu'il aurait pu écrire et qui n'est autre qu'une part de lui-même, désenfouie, réactivée. 

orientations politiques et esthétiques, le choix des films, des personnes à filmer mais c'est aussi dans un même mouvement pouvoir être suffisamment dissemblables pour devenir complémentaires. Pouvoir s'enrichir de la vision de l'autre, bénéficier de compétences différentes des siennes. composantes. La vie (commune) est nécessairement à la base de la recherche-création mais non, toujours, comme matière première (auto)biographique. Vivre ensemble, c'est aussi interagir avec le monde extérieur, faire des choix politiques en commun. S'accorder sur des choix de vie et de société. S'accorder, par exemple, sur l'importance que revêt aujourd'hui la contradiction entre la société et la nature.

18 Nous avons décidé de réaliser ensemble plusieurs films en agriculture biologique. Où sommes-nous dans ces films ? S'il est certain que nous ne sommes pas les paysans chercheurs que nous filmons, nous interagissons avec eux. Nous sommes en accord avec leur ligne de vie et de travail. Ils ont des choses à dire et à montrer, et notre travail consiste à les faire advenir dans les meilleures conditions possibles. Ces choses ont été ressenties, pensées, tournées et retournées dans le cerveau des paysans chercheurs avec lesquels nous avons entrepris de travailler. Il en résulte, pour celui ou celle qui parle, une parole aussi assurée de ses assises et fondations que peut l'être l'architecte d'un édifice bien conçu et bien construit. Il s'agit d'une parole arrivée à maturité comme un fruit que l'on peut savourer à l'instant. Et c'est bien ici que cette parole a été cultivée : dans ce champ de triticale ou dans ce champ de seigle. Bien entendu, elle n'est pas née d'ellemême ni de son environnement immédiat, elle s'est également nourrie d'apports extérieurs, parfois lointains. Mais ces apports ont été assimilés et interprétés en fonction des conditions de culture propres à chaque ferme. Ce qui réussit chez l'un ne réussit pas nécessairement chez l'autre, vivraient-ils à quelques kilomètres de distance. Le sol peut être très différent d'une parcelle à l'autre, parfois sur la même ferme. 
19 Nous l'avons compris très vite : un paysan chercheur ne fait usage ni de prêts à penser ni de prêts à planter. Il n'applique pas de recettes. Il sait que la nature est complexe et il refuse de réduire cette complexité sous la botte chimique qui est supposée pourvoir à tout. Il ne cherche pas à dompter la supposée dangereuse nature, il prend le parti de l'accompagner en comprenant de mieux en mieux les contradictions qui la font se développer en dehors de tout schéma manichéen.

Il n'y a par conséquent rien de dogmatique dans les discours qui nous sont tenus. Cette parole qui affirme des orientations, des positions, cette parole sûre d'elle-même se met en même temps en jeu dans des hypothèses de recherche et de travail qu'il s'agit toujours de vérifier sur le terrain. Il y a souvent des possibles, parfois des probabilités, jamais des certitudes. Ce n'est pas qu'elle doute de ses orientations et positions : ce sont ces dernières qui l'amènent à construire des hypothèses.

21 Une parole enracinée, ce n'est pas seulement du sens, des concepts. Ce sont aussi des percepts et des affects. C'est ce mélange, comme on le dit pour les associations de cultures, qui lui donne sa charge émotive et la rend immédiatement partageable et appropriable. Ici les émotions ne sont pas utilisées pour manipuler les idées ni les idées pour manipuler les émotions. Les émotions et les idées ont grandi ensemble. Elles s'entremêlent continûment et s'affinent constamment au contact les unes des autres.

Notre travail de cinéastes ? Susciter cette parole, la libérer, la capter dans son surgissement. La croiser avec d'autres paroles, saisies dans les mêmes conditions. Croiser les paroles ? Pas seulement. Les faire interagir. Elles se renforcent, se contredisent, se complètent. Organiser par le montage des rencontres qui n'ont pas encore eu lieu dans la réalité. C'est à cela, aussi, que sert le montage dans un film: transformer des rencontres virtuelles en rencontres réelles. Anticiper la réalité.

Donner tant d'importance à la parole, ne serait-ce pas de la radio ? Mais non. D'abord, parce qu'une radio digne de ce nom ancre la parole dans des environnements sonores qui suscitent des images visuelles chez l'auditeur. La radio n'est jamais seulement une radio oreille, comme un sens n'est jamais seul et interagit toujours avec les autres dans des proportions variables selon les situations. Les différentes façons de pratiquer la radio favorisent plus ou moins ces interactions mais elles existent toujours.

Mais il y a un autre point qui mérite d'être rappelé et exploré : le film ancre la parole au lieu de son émission. Ce lieu est double : il y a le corps de celui qui émet cette parole mais il y a aussi l'environnement auquel cette parole se rattache. Cet environnement est plus vaste que le territoire occupé par la ferme. Il s'inscrit dans un paysage, à la fois physique et mental, qu'il contribue à remodeler.

L'un et l'autre peuvent être saisis simultanément dans les mêmes cadres. Nos cadres expriment un double choix: affirmer la primauté de l'environnement sans effacer l'homme, affirmer la primauté de l'homme sans effacer l'environnement. L'homme et son environnement se situent à égalité, sans que le premier cherche à dominer le second : simplement le sentir, le comprendre. Mais ce simplement est aussi vaste que la recherche, toujours en cours, jamais achevée.

26 Notre démarche vise aussi à saisir ce qu'il y a de sensible dans la relation de nos « acteurs " (acteurs de leur vie avant d'être acteurs de nos films) à leur environnement. Cette relation sensible se manifeste dans les discours mais aussi dans la gestuelle qui les accompagne.Voyez par exemple Lionel Caudy dans son champ de seigle au cours d'un tour de plaine où il confronte ses hypothèses de travail aux questions et 
expérimentations d'autres paysans (L'autonomie paysanne, 2013). Tout en parlant, il se met à caresser les épis. La caresse fait écho à la rigueur d'un discours tout en questionnements. Sans cet amour de la nature qui se matérialise ici dans la douceur de ce geste, il n'y aurait pas de pensée, du moins pas ce type de pensée.

\section{Couples}

27 Nous formons un couple qui filme souvent - hasard ou nécessité ? - d'autres couples. Voici Entredeux (2015). Artistes et artisans d'art, Bénédicte Magnin Robert et Jean-Luc Bernard sont devant notre caméra. Ils vivent ensemble et, s'ils ont chacun leur atelier, leur travail est sans cesse sous le regard de l'autre. Ils se retrouvent aussi ensemble pour des créations en commun. Nous nous sommes embarqués avec eux pour la réalisation d'un film, non sur les secrets de la création, mais sur ses processus. Un couple en filme un autre. Dès la première projection, des spectateurs font remarquer à quel point les interactions des deux couples sont sensibles à l'écran.

À quoi cela tient-il ? D'abord, au fait que nous nous connaissons dans la vie et que nous sommes amis. Nous sommes parvenus à un degré d'intimité qui nous permet d'échanger sur les processus de création. Ils savent que nous sommes sensibles à leur démarche et que nous la comprenons. Une relation de confiance réciproque s'est établie. Lorsque le film s'engage, Bénédicte et Jean-Luc savent que nous n'allons pas les filmer en simples spectateurs mais en acteurs d'un autre processus de création, cinématographique, auxquel ils sont eux-mêmes sensibles et auquel ils adhérent (nous avions déjà réalisé un autre film avec Jean-Luc, Osier vole !, et ils ont vu tous nos films). Ils peuvent alors présenter leur travail de l'intérieur dans une relation intersubjective avec la caméra. La caméra ? Une caméra habitée d'une façon qui leur parle. Une caméra qui ne fait pas écran entre leur travail et le notre. Un monde imaginaire s'épanche dans un autre et réciproquement. Nous allons filmer leurs objets - des luminaires, des sculptures - et tenter de trouver les mots qui traduisent ce que nous ressentons à leur approche.

Ce n'est pas seulement filmer à deux, c'est filmer en couple, dans une dynamique de couple, qui permet de capter une autre dynamique de couple. La dynamique de couple conditionne parfois l'existence et le développement d'une activité. Voici Perrine et Charles Hervé Gruyer que nous avons filmé pour L'horizon des possibles (2017). Figures françaises de la permaculture parmi les plus reconnues, les Gruyer sont fortement médiatisés. Devant notre caméra, ils mettent en relation l'histoire de leur ferme avec l'histoire de leur couple. Bien sûr, nous les avons incités à le faire. Mais aurions-nous obtenu des interactions aussi profondes si nous n'avions pas formé nous-mêmes un couple sensible au fait qu'ils en formaient un ? À voir les autres films où ils apparaissent, on peut en douter.

\section{Artisanat}

Le mode de production artisanal de nos films nous rapproche de ceux que nous filmons (paysans bio, artistes, artisans d'art). Même si nos activités sont différentes, nous avons en commun le désir de maitriser l'ensemble des processus en jeu dans ces activités. Cela nous met sur un pied d'égalité et introduit entre nous une connivence dont les traces se repèrent aisément dans nos films. 
Nous filmons avec de petites caméras, amateurs ou semi professionnelles. Pour En amour et Premiers mois, nous avons choisi une caméra-corps susceptible de s'intégrer au mieux aux mouvements de la vie. Il n'était pas question de la placer sur un pied. Il n'était pas non plus question de la fixer sur l'épaule. Nous la tenions à la main, elle était un prolongement de notre regard. Parfois nous filmions à partir d'un autre point du corps, sans cadrer. Ce sont les sensations que nous éprouvions au moment de filmer qui nous dictaient de tels choix.

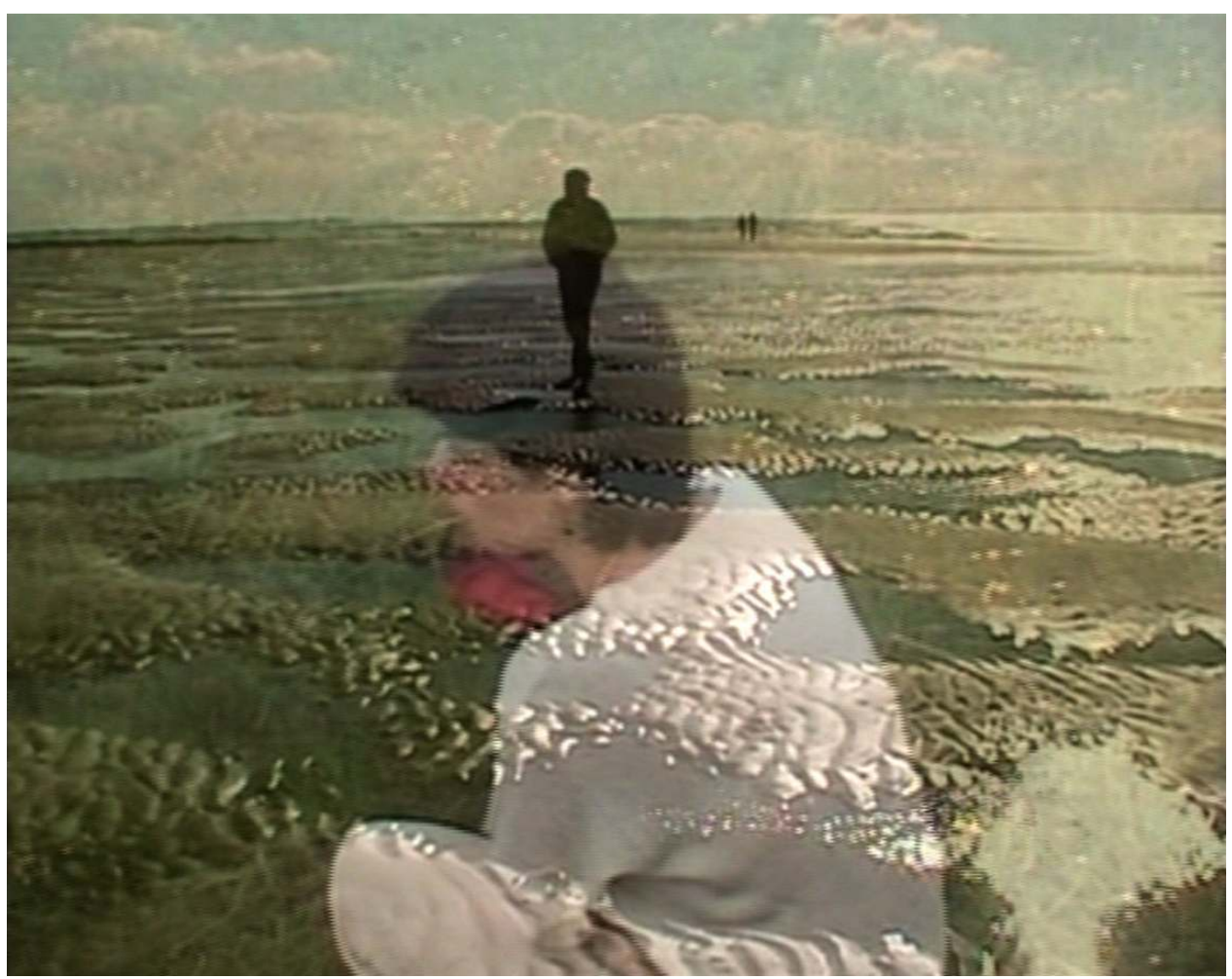

En amour, 2001

Dans les films qui ne relèvent pas de l'entrefilmage, nous recourons fréquemment au pied. Pas seulement pour les entretiens. Pour filmer des ciels, des paysages, des objets. Le mouvement de la vie se déplace alors dans ce que nous filmons comme si la notre était suspendue au déroulement du plan. Le micro est fixé le plus souvent sur la caméra. Nous ne perchons pas. Parfois, l'un(e) de nous tient le micro à la main tandis que l'autre filme. Il nous arrive aussi d'utiliser le micro-cravate pour libérer la caméra.

Nous cherchons dans tous les cas une économie de moyens techniques qui nous rapproche encore une fois de la littérature. Caméra stylo ? Oui, si l'on entend par là présence au monde.

34 Nous publions ci-après le texte que nous avons présenté au colloque "Lettre filmée, film-lettre, lettre de cinéaste » (Université Paris 8 et Institut National d'histoire de l'art, 12 et 13 mai 2014) dont les actes sont restés inédits. Ce texte concerne En amour (2001) qui est le film fondateur de notre couple et de notre travail ultérieur en commun.

\section{S'entrefilmer}


Je n'admets qu'on propose à l'homme que des objets de jouissance, d'exaltation, de réveil.

Francis Ponge. fondamental encore. Les frontières identitaires se lézardent et tendent à disparaître. On 
se déplace de l'un à l'autre et c'est dans cet entre-deux qui s'ouvre de façon vertigineuse qu'un nouveau champ d'exploration se constitue où se profile la construction d'une identité inconnue : $1+1=3$. L'amour donnerait lieu en quelque sorte à un profond séisme qui ferait éclater les cloisonnements des identités respectives, en créant ainsi un mouvement capable de générer une nouvelle forme identitaire, plus riche, plus intense, démultipliée par l'interaction des deux autres.

Le film va se calquer sur ce que la relation amoureuse comporte de suspens et d'imprévisible. On ne sait pas ce qui va arriver ou plutôt on ne sait pas ce qui va se vivre. On ne sait pas non plus comment va se manifester le désir de filmer dans ce qui va se vivre. Si les lieux de tournage sont avant tout des «lieux de vie ", comme nous le signifions dans le film, ce n'est pas pour autant que l'on filme en tout temps, en tout lieu. Encore faut-il que s'impose le désir de filmer, que le désir de filmer soit une composante du désir de vivre, autrement dit qu'il interagisse de façon positive avec la réalité vécue hors cinéma.

L'imprévu du tournage n'est pas de même nature que celui du montage. Au tournage, il s'agit de vivre le film dans le mouvement même de la vie, à son rythme et dans ses aléas. Il est trop tôt encore pour adopter un principe de construction. Ce serait vouloir en imposer à la vie, ce serait vouloir s'imposer à la vie en tant qu'auteur (créateur) de cette vie. Mais il est déjà possible et même nécessaire de prévoir un dispositif de filmage fondé sur le refus de toutes les formes de séparation, à commencer par celles issues de la division sociale et technique du travail. Nous filmerons réellement à deux. Nous ne nous placerons pas dans la posture de deux co-réalisateurs dirigeant une équipe de prise de vue dirigée elle-même par un chef opérateur. Il n'y aura pas d'opérateur à part nous. D'où le problème de la circulation de la caméra.

En fonction d'une situation et de son évolution, en fonction de l'implication mouvante de chacun dans cette situation, en fonction des angles et des cadres possibles, il faudra se passer la caméra.

Comment effectuer cette transmission sans que l'un ordonne à l'autre de la faire ? Cela suppose évidemment une communication intense entre les deux filmeurs. Au montage, la vie continue avec le matériau recueilli au tournage. Ce qui fut vécu au tournage, cinématographiquement parlant se résume à présent à ce matériau. Il est temps d'inventer un principe d'organisation du matériau, susceptible d'accueillir encore de l'imprévu. Mais l'imprévu va naître ici de l'organisation du matériau lui-même, c'est-àdire du montage.

Tournage et montage ont toutefois en commun le principe de discontinuité. Mais la discontinuité au tournage est liée aux moments de la vie où le désir de filmer se matérialise (par le filmage de situations saisies dans leur durée réelle ou de façon plus fragmentaire, éclatée) et la discontinuité au montage à l'organisation du matériau. Le fait que le tournage soit suspendu à deux formes d'imprévisibilité (celle de la vie, celle du désir de filmer) n'exclut nullement une forme de scénarisation ouverte : un canevas de vie, en quelque sorte. Nous décidons de voyager à partir de la nécessité profondément ressentie de croiser nos enfances. Croiser des lieux, rencontrer des gens, connus de l'un, inconnus de l'autre, et les (re)découvrir ensemble comme si nous ne les avions jamais vus. Le voyage pourra s'arrêter au square Saint-Lambert, dans le quinzième arrondissement de Paris. Un ticket de métro est suffisant pour l'accomplir. Il pourra nous emmener un peu plus loin, à Gurgy-la-Ville, dans la Bourgogne profonde. Un peu plus loin encore : à Perpignan et ses alentours. Ou encore à Lisbonne, où il est question d'un projet de film, 
Tous ces lieux ont été choisis en commun, leur visite a été programmée, mais nous ignorons tout du regard nouveau qui naîtra de leur (re)découverte. De même l'un au moins d'entre nous connaît-il les personnes que nous allons rencontrer puisqu'elles font partie de sa famille. Mais nous ignorons (inégalement selon les rapports plus ou moins étroits ou distendus avec chacune d'entre elles) leur état d'esprit du moment, la façon dont elles vont réagir à notre présence commune, le type de situations qui va se présenter, le type de relations que nous allons établir avec elles. La programmation comporte donc une large part d'imprévisible que nous n'aurions voulu réduire pour rien au monde.

47 Au montage, il s'agit toujours de partir de ces interactions filmées pour en construire de nouvelles, celles qui naîtront des images entre elles, du travail du film. Le montage ne répondra pas au principe de la transparence ni à celui de la continuité narrative. Il affirme au contraire son caractère fragmentaire, assemble, disjoint, divise, superpose les images les unes avec les autres. Penser l'intervalle, l'interaction des plans ou des séquences entre elles en assumant la discontinuité, l'hétérogène. Il fait apparaître d'autres combinaisons, d'autres liens possibles entre les scènes (le vrai montage commence quand on ne sait pas ce qui va arriver, écrivait Jean-Daniel Pollet dans L'entreVues). Le montage devient une construction poétique qui ne se soumet à aucune règle héritée de la narrativisation ou de la discursivité. Il se libère des significations posées a priori pour laisser place à un imaginaire vécu où l'inconscient joue un rôle majeur.

Des lieux, des saisons se succèdent, des paysages changeants se rencontrent, brisant toute continuité narrative, tout repère spatio-temporel pour participer à la construction filmique d'un espace-temps inédit, celui de l'amour. Tout ce désordre apparent permettrait de créer avec les moyens du cinéma un ordre nouveau, capable d'exprimer quelque chose qui n'aurait pas eu lieu sans le film, plus proche du sensible. Le sensible ne se laisse pas réduire ici à un moyen d'information pour la perception, il constitue la base même de la construction poétique du film.

Je prendrai l'exemple de ma tante Marie. Une relation forte mais laissée en friches pour diverses raisons depuis plusieurs années. Nous apprenons qu'elle a quitté sa maison du Boulou et qu'elle vit maintenant dans une maison de retraite. Ce fait, parfaitement prévisible, n'a rien d'un événement. Ma tante Marie a 92 ans au moment du tournage, en 2001. Dans nos sociétés, il est statistiquement commun que l'on vieillisse en maison de retraite et que l'on meure à l'hôpital. Le sort de ma tante Marie ne déroge pas à l'ordre présumé normal de la société. Mais elle se révolte contre cet état de choses, nous dit Milou, le fils qui n'a jamais accepté qu'elle remplace sa mère auprès de son père. Nous décidons d'aller la voir. La caméranous accompagne, mais nous ne savons pas si nous allons filmer. Tout dépendra de la situation qui va se créer là-bas. Comment va-t-elle m'accueillir après tout ce temps passé sans lui donner la moindre nouvelle ? Comment vat-elle accueillir Catherine, qu'elle n'a jamais vue ? Elle ne sait même pas que nous vivons ensemble. Autre inconnue : Milou nous a dit qu'elle n'avait plus toute sa tête. Va-t-elle seulement me reconnaître ou bien me prendre pour quelqu'un d'autre qui me ressemblerait vaguement? Ou me demander qui je suis ? Ou se désintéresser de notre présence?

50 Milou avait (presque) tout faux. Marie a suffisamment de tête pour aimer et se révolter. La relation affective est immédiate, le passé intensément présent. Aucune question sur la légitimité du couple que nous formons avec Catherine. Elle ressent le flux amoureux qui circule entre nous et cela lui suffit. Sans que nous nous soyons donné le mot, Catherine à 
commencé à filmer. Ce qui nous décide à continuer, c'est le rôle que Marie confère d'emblée à une caméra que nous ne tentons jamais de dissimuler, aussi petite soit-elle. Le rôle d'un témoin dans le conflit qui l'oppose à Milou et à son discours, raisonnable en apparence et lâche dans les faits. Elle va s'adresser à elle à plusieurs reprises, et au spectateur virtuel qui se trouve derrière (pour l'instant, il s'agit de Catherine ou de moi), pour démonter le discours imperturbable de Milou, par les mimiques et la gestuelle davantage encore que par les mots. Par son attitude ludique et théâtrale, elle renvoie le comportement et le discours de Milou à ce qu'ils sont en réalité: de la représentation. La caméra passe progressivement du statut de témoin à celui d'actrice. La situation, dans son cours imprévisible, devient une scène à transformations multiples où se croisent, se font et se défont les espaces visuels et sonores.

Nous avons beaucoup filmé ce jour-là, mais nous ne savons pas si ce que nous avons filmé trouvera sa place dans le film. N'y avait-il pas dans ce continuum audiovisuel, où durée subjective et durée réelle coïncidaient si étroitement, l'embryon d'un autre film qu'il nous fallait développer selon l'axe qui lui était propre? Ne s'agissait-il pas d'un autre sujet, traité dans un style très différent de celui que nous avions adopté jusqu'ici, où la durée réelle est recomposée par la durée subjective ? Mais en commençant à monter cette séquence avec le matériau recueilli dans la famille de Catherine sur la fabrication du miel, il nous parut évident que le croisement de nos enfances atteignait dans ce montage son point culminant. Il y aurait des moments de montage liés davantage à la réalité vécue " sur le terrain " et d'autres moments à la réalité d'un imaginaire recomposé cinématographiquement. Ce qui allait donner son unité à notre film, ce n'était ni une unité de style, ni une unité dans les méthodes d'approche de la réalité visible, mais une succession d'états subjectifs vécus avec intensité. La restitution de l'état l'emportait sur tout autre préoccupation, narrative ou discursive. C'est en quoi notre démarche cinématographique se rapprochait de la démarche poétique : refuser la réduction de l'état à du récit comme à du discours. L'état subjectif - qu'il soit émotif et/ ou cognitif - peut naître des situations les plus diverses, celles que la vie, où la programmation ne peut éliminer l'imprévisible, ménage précisément.

Une situation peut être imprévisible en elle-même ou le devenir alors qu'elle était programmée dans les moindres détails. Le cinéma sera en mesure d'intégrer toutes les situations possibles s'il ne tente pas de rendre homogène ce qui est hétérogène dans la vie. Ce qui fait l'unité d'un film, c'est la relation qu'une subjectivité entretient avec ce qu'elle vit et ce qu'elle filme, aussi divers soit-il. Le sujet d'un film n'est pas dans la situation mais dans la subjectivité qui vit et filme cette situation. S'il se propose d'accueillir la vie dans l'ensemble de ses surgissements, un film sera nécessairement fait de sauts, de ruptures, de changements de registre.

L'état amoureux crée une temporalité qui lui est propre et qui résiste au temps biologique comme au temps social. Par là-même, il invente des espaces où intérieur et extérieur ne s'opposent plus. Le voyage est avant tout déplacement identitaire. La nouvelle identité se construit à travers les paysages revisités, que chacun a vécu dans son enfance avec intensité, et maintenant re-vécus ensemble, transformés. Mais le projet de croiser nos enfances au tournage devait trouver son prolongement au montage.

Comment construire, au montage, un espace-temps qui correspondrait au surgissement d'une nouvelle identité ? Il y eut d'abord le refus de la linéarité et de toute forme de continuité narrative ou discursive. Le film serait fondé sur la discontinuité. Nous n'impulserions au film d'autre progression que celle qui va de la séparation à des formes 
d'interaction de plus en plus fortes. Très vite s'est imposé à nous le principe du croisement des espaces visuels et sonores avec, pour technique majeure, un usage particulier de la surimpression. Il était impossible de préfigurer ce que deviendraient ces surimpressions d'espaces avant de les expérimenter.

C'était là, nous semblait-il, la forme la plus adéquate à ce que nous vivions. L'imprévisible continuait d'avoir cours sur le logiciel de montage. Que se passe-t-il, en effet, avec la surimpression? On part d'espaces visuels et sonores, reconnaissables et identifiables en tant que tels et, en surimprimant ces espaces, on aboutit à la création d'un nouvel espace issu du mélange des éléments préexistants. S'il est possible d'en reconnaitre encore certains aspects, c'est la relation de transformation avec les autres éléments préexistants qui nous importe désormais. C'est le processus de transformation lui-même qui se trouve au centre de notre attention. Décomposition et recomposition des formes. Mais la recomposition des formes n'est pas la reconduction des formes préexistantes. Elle n'y fera jamais retour. Le processus de transformation est irréversible et imprévisible. Il n'y a plus d'autre temporalité que celle issu du processus lui-même. Un espace-temps inédit se construit peu à peu.

Ainsi la surimpression correspond-elle très précisément à la relation amoureuse, telle que nous la concevons. Figure centrale de la rencontre d'images, du surgissement imprévisible d'espaces et de temporalités nouvelles, à l'image de la transformation identitaire qui ébranlait nos vies à ce moment là. "Les rencontres d'images seraient comme la respiration du film, des images qui se reconnaitraient, se mêleraient amoureusement, basculeraient les unes dans les autres pour en faire naître d'inconnues. Impossible de préfigurer ce qu'elles seront ». Par la surimpression, les images se transforment les unes par les autres, inventent des formes nouvelles sans plus se laisser enfermer dans des formes aux contours bien définis, espèrent créer de nouveaux rapports à l'imaginaire. Tout comme la relation amoureuse, la surimpression assemble, transforme, recompose.

57 Les images s'enlaceraient comme des corps sans plus jamais se laisser enfermer dans des formes aux contours bien définis, il n'y aurait plus de frontière d'un corps à l'autre, d'un paysage à l'autre, du désir à la pensée.

\section{Filmographie}

En amour (2001), La peute bête (2004), Premiers mois (2005), Tout un fromage de Langres (2006), L'âme du cochon (2006), Couleurs en lumière (2007), Langue de bois (2007), La patine des siècles (2007), Osier vole! (2007), Les bijoux indiscrets (2007), Par-dessus la tête (2007), Au fil du temps (2007), Au grès des formes (2007), Vous n'y couperez pas (2008), Du côté de Montsaugeon (2008), L'écorce des pierres (2009), Un autre horizon (2010), Le peuple des écorces (2013), L'autonomie paysanne (2013), Langres, Diderot et nous... (2014), Entre deux (2015), L'horizon des possibles (2017). 


\section{BIBLIOGRAPHIE}

Livres-DVD : « Les yeux au bout des doigts » (2010), « Langres, Diderot et nous... » (2015).

G. Leblanc, « Un cinéma du subjectif », Cahier Louis-Lumière n ${ }^{1}$, août 2003.

C. Guéneau, « Filmer en amour », Cahier Louis-Lumière n¹, août 2003.

G. Leblanc, « De l'expression subjective directe », Cahier Louis-Lumière nº 8 , juillet 2011.

C. Guéneau, «La mise en “je” du film », Cahier Louis-Lumière n8, juillet 2011.

C. Guéneau et G. Leblanc : « La rencontre amoureuse et les déplacements de l'espace-temps cinématographique », dans Cinéma et voyage, sous la direction de René Gardies, Paris,

L'Harmattan, 2007.

G. Leblanc, Presque une conception du monde, Grane, Créaphis, 2007.

Site : http://www.mediascreationrecherche.fr

\section{NOTES}

1. Odile Richard-Pauchet écrit dans le numéro 51 des Recherches sur Diderot et sur l'Encyclopédie (2016) : « (...) Un certain nombre de pages nous ont particulièrement marquée par leur caractère poétique : la sensibilité de l'écrivain aux états météorologiques et aux grands ciels... ; sa sensibilité aussi à la qualité minérale du site langrois dont sa pensée matérialiste et un certain nombre d'images philosophiques resteront marquées (deux éléments constitutifs de la scénographie du film Langres, Diderot et nous...).

\section{RÉSUMÉS}

Les implications du travail à deux dans le cas de figure où $1+1=3$. Comment se construit cette troisième personne qui se nourrit des deux autres sans s'y réduire ? Par l'instauration d'un dialogue qui excède les limites de l'un.e et de l'autre. Le questionnement théorique et pratique ouvre sur l'analyse du premier film réalisé en commun: En amour (2001).

The implications of two people working together in the case when $1+1=3$. How does this third person develop its own self while it feeds on the two others without being reduced to them? By establishing a dialogue that goes beyond the limits of the one and the other. The theoretical and practical questioning opens out onto the analysis of the first film made together: En amour (2001). 
INDEX

Mots-clés : interactions, entrefilmage, intime, amour, autofiction

\section{AUTEURS}

\section{CATHERINE GUÉNEAU}

Réalisatrice et chargée de recherche dans le domaine du cinéma et des technologies numériques. Ensemble, ils ont créé l'association Médias Création Recherche (production d'essais et de films documentaires) et co-réalisé entre autres : En amour, Premiers mois, Gestes d'art, L'âme du cochon, L'écorce des pierres, Un autre horizon, L'autonomie paysanne, Langres, Diderot et nous, l'horizon des possibles.

\section{GÉRARD LEBLANC}

Professeur émérite des universités, écrivain et réalisateur. Derniers livres publiés : Trajectoires (Editions de L'oeil), Presque une conception du monde (Editions Créaphis), Les yeux au bout des doigts (Médias Création Recherche). 